SUPPORTING INFORMATION

\title{
TRIFLIC ANHYDRIDE-A MILD REAGENT FOR HIGHLY EFFICIENT SYNTHESIS OF 1,2-BENZISOXAZOLES, ISOXAZOLO AND ISOTHIAZOLO QUINOLINES WITHOUT ADDITIVE OR BASE
}

\author{
Rajesh G. Kalkhambkar, ${ }^{1}$ and H. Yuvaraj, ${ }^{2}$ \\ ${ }^{1}$ Department of Chemistry, Karnatak University's Karnatak Science College, Dharwad \\ Karnatak 580001, India \\ ${ }^{2}$ School of Display and Chemical Engineering, Yeungnam University, Gyeongsan, \\ Gyeoungbuk 712-749, Republic of Korea
}




\section{EXPERIMENTAL (GENERAL)}

The reagents employed were of high purity commercial samples which were used as received (Fischer, Merck and Sigma Aldrich). Reactions were carried out in oven-dried RB flask. Column chromatography was performed on silica gel (200-400 mesh). TLC was performed on alumina silica gel $60 \mathrm{~F}_{254}$ (Fischer) detected by UV light $(254 \mathrm{~nm})$ and iodine vapors. The melting points were determined by open capillaries on a Buchi apparatus and are uncorrected. The IR spectra were recorded on a Nicolet-Impact-410 FT-IR spectrometer, using $\mathrm{KBr}$ pellets. ${ }^{1} \mathrm{H}$ NMR and ${ }^{13} \mathrm{C}$ NMR spectra were recorded on a Bruker AC-300F, $300 \mathrm{MHz}$, spectrometer in DMSO- $d_{6}$ using TMS as an internal standard with ${ }^{1} \mathrm{H}$ resonance frequency of $300 \mathrm{MHz},{ }^{13} \mathrm{C}$ resonance frequency of $75 \mathrm{MHz}$. GC analyses were performed on Nucon 5700 series Gas chromatograph. GC-MS analyses were performed on Shimadzu 2010 series mass selective detector instrument. Elemental analysis was carried out by using Heraus CHN rapid analyzer. All the compounds gave $\mathrm{C}, \mathrm{H}$ and $\mathrm{N}$ analysis within $\pm 0.4 \%$ of the theoretical values. Dry DCM and EDC were obtained from commercial source by the standard procedure. ${ }^{[27]}$

\section{Typical procedure for the synthesis of 1,2-benzisoxazole using triflic anhydride}

The desired 2-hydroxyaryl aldoxime or ketoxime $(2.0 \mathrm{mmol})$ in $5 \mathrm{~mL}$ dry DCM was taken in an oven-dried RB flask. To the reaction mixture was added drop wise triflic anhydride $(2.0 \mathrm{mmol})$ in DCM under nitrogen for 15 minutes. The reaction mixture was stirred at RT and the progress of the reaction was monitored by TLC and GC-MS (Table 1). After completion of reaction, the contents were poured to crushed ice $(100 \mathrm{~mL})$ and neutralized with $10 \% \mathrm{NaHCO}_{3}$ solution $(20 \mathrm{~mL})$ and extracted with DCM (30-40 $\mathrm{mL}$ ). The pure products were obtained by column chromatography with hexane-ethyl acetate mixture (80:20). All the 1,2-benzisoxazole derivatives were characterized by GC-MS, ${ }^{1} \mathrm{H},{ }^{13} \mathrm{C}$ NMR and by elemental analysis and the results are compared with authentic samples. 


\section{Characterization data of various substituted 1,2-benzisoazole derivatives}

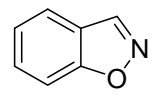

\section{1,2-Benzisoxazole (1) ${ }^{1,3,4}$}

Yield: $230 \mathrm{mg}$ (96\%); colorless liquid. IR (KBr): 3045, 2919, 1620, 1513, 1463, 1407, 1246, 1028, 822, $712 \mathrm{~cm}^{-1} .{ }^{1} \mathrm{H}$ NMR $\left(300 \mathrm{MHz}, \mathrm{DMSO}-d_{6}\right): \delta=7.19(\mathrm{~m}, 4 \mathrm{H}), 8.43(\mathrm{~s}, 1 \mathrm{H}) .{ }^{13} \mathrm{C} \mathrm{NMR}(75 \mathrm{MHz}$, DMSO- $\left.d_{6}\right): \delta=161.0,143.8,135.2,123.9,121.0,120.2,110.4$. GC-MS: m/z 119 [M] $]^{+}$. Anal. Calc. For $\mathrm{C}_{7} \mathrm{H}_{5} \mathrm{NO}: \mathrm{C}, 70.58 ; \mathrm{H}, 4.23 ; \mathrm{N}, 11.76 \%$ found: $\mathrm{C}, 70.52 ; \mathrm{H}, 4.21 ; \mathrm{N}, 11.70 \%$.

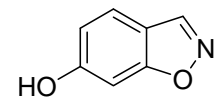

\section{6-Hydroxy-1,2-benzisoxazole (2) ${ }^{1}$}

Yield: 260 mg (93\%); colorless liquid. IR (KBr): 3250, 3062, 2957, 2930, 1628, 1604, 1576, 1541, 1308, 1141, $696 \mathrm{~cm}^{-1} .{ }^{1} \mathrm{H}$ NMR (300MHz, DMSO- $\left.d_{6}\right): \delta=4.65(\mathrm{~s}, 1 \mathrm{H}, \mathrm{OH}), 6.80(\mathrm{~m}, 2 \mathrm{H}), 7.20(\mathrm{~m}$, $1 \mathrm{H}), 8.44(\mathrm{~s}, 1 \mathrm{H}) .{ }^{13} \mathrm{C}$ NMR $\left(75 \mathrm{MHz}, \mathrm{DMSO}-d_{6}\right): \delta=156.1,152.1,148.8,135.3,128.1,125.7$, 118.4. GC-MS: m/z 135 [M] ${ }^{+}$. Anal. Calc. For $\mathrm{C}_{7} \mathrm{H}_{5} \mathrm{NO}_{2}$ : C, 62.22; H, 3.73; N, 10.37\% found: C, 62.26; H, 3.70; N, $10.32 \%$.

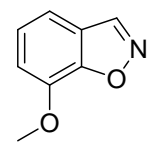

\section{7-Methoxy-1,2-benzisoxazole (3) ${ }^{1,2}$}

Yield: 288 mg (90\%); yellow liquid. IR (KBr): 3051, 3031, 1626, 1582, 1530, 1474, 1411, 1285, 1229, $794 \mathrm{~cm}^{-1} .{ }^{1} \mathrm{H}$ NMR (300MHz, DMSO- $\left.d_{6}\right): \delta=3.78\left(\mathrm{~s}, 3 \mathrm{H}, \mathrm{OCH}_{3}\right), 6.75(\mathrm{~m}, 2 \mathrm{H}), 7.15(\mathrm{~m}, 1 \mathrm{H})$, $8.47(\mathrm{~s}, 1 \mathrm{H}) .{ }^{13} \mathrm{C}$ NMR $\left(75 \mathrm{MHz}, \mathrm{DMSO}-d_{6}\right): \delta=152.1,148.8,145.3,134.6,128.1,124.9,117.1$, 58.9. GC-MS: $\mathrm{m} / \mathrm{z} 149[\mathrm{M}]^{+}$. Anal. Calc. For $\mathrm{C}_{8} \mathrm{H}_{7} \mathrm{NO}_{2}$ : C, 64.42; $\mathrm{H}, 4.73 ; \mathrm{N}, 9.39 \%$ found: $\mathrm{C}, 64.38$; $H, 4.70 ; N, 9.35 \%$. 


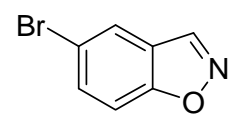

\section{5-Bromo-1,2-benzisoxazole (4) ${ }^{1}$}

Yield: 375 mg (88\%); light brown liquid. IR (KBr): 3057, 1625, 1593, 1573, 1516, 1394, 1310, 1090, 821, $714 \mathrm{~cm}^{-1} .{ }^{1} \mathrm{H}$ NMR (300MHz, DMSO- $\left.d_{6}\right): \delta=7.32(\mathrm{~m}, 3 \mathrm{H}), 8.50(\mathrm{~s}, 1 \mathrm{H}) .{ }^{13} \mathrm{C} \mathrm{NMR}$ $\left(75 \mathrm{MHz}, \mathrm{DMSO}-d_{6}\right): \delta=162.4,142.5,134.2,124.6,122.1,122.1,112.4$. GC-MS: m/z $196[\mathrm{M}]^{+}$, $198[\mathrm{M}]^{+}$. Anal. Calc. For $\mathrm{C}_{7} \mathrm{H}_{4} \mathrm{BrNO}$ : C, 42.46; H, 2.04; N, 7.07\% found: $\mathrm{C}, 42.42 ; \mathrm{H}, 2.01 ; \mathrm{N}, 6.98$ $\%$.

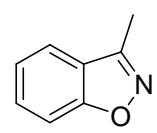

\section{3-Metyl-1,2-benzisoxazole (5) ${ }^{1,2}$}

Yield: 260 mg (96\%); colorless liquid. IR (KBr): 3037, 2919, 1614, 1597, 1527, 1405, 1323, 813 $\mathrm{cm}^{-1} .{ }^{1} \mathrm{H}$ NMR $\left(300 \mathrm{MHz}, \mathrm{DMSO}-d_{6}\right): \delta=2.38\left(\mathrm{~s}, 3 \mathrm{H}, \mathrm{CH}_{3}\right), 7.29(\mathrm{~m}, 4 \mathrm{H}) .{ }^{13} \mathrm{C}$ NMR $(75 \mathrm{MHz}$, DMSO$\left.d_{6}\right): \delta=160.3,152.1,130.8,126.1,122.1,121.3,112.1,16.5$. GC-MS: m/z 133 [M] ${ }^{+}$. Anal. Calc. For $\mathrm{C}_{8} \mathrm{H}_{7} \mathrm{NO}$ : C, 72.16; $\mathrm{H}, 5.30 ; \mathrm{N}, 10.52 \%$ found: $\mathrm{C}, 72.15 ; \mathrm{H}, 5.28 ; \mathrm{N}, 10.49 \%$.

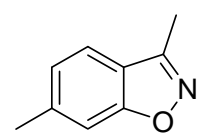

\section{3, 6-Dimethyl-1,2-benzisoxazole (6) ${ }^{1,2}$}

Yield: 290 mg (90\%); light yellow liquid. IR (KBr): 3046, 3018, 2958, 1622, 1542, 1421, 1315, 1250, 850, $728 \mathrm{~cm}^{-1} .{ }^{1} \mathrm{H}$ NMR $\left(300 \mathrm{MHz}, \mathrm{DMSO}-d_{6}\right): \delta=2.30\left(\mathrm{~s}, 3 \mathrm{H}, \mathrm{CH}_{3}\right), 2.48\left(\mathrm{~s}, 3 \mathrm{H}, \mathrm{CH}_{3}\right), 7.23$ (m, 3H). ${ }^{13} \mathrm{C}$ NMR $\left(75 \mathrm{MHz}\right.$, DMSO-d $\left.d_{6}\right): \delta=160.3,153.1,132.8,125.1,121.4,121.1,110.2,18.6$, 16.8. GC-MS: m/z 147 [M] $]^{+}$. Anal. Calc. For $\mathrm{C}_{9} \mathrm{H}_{9} \mathrm{NO}: \mathrm{C}, 73.45 ; \mathrm{H}, 6.16 ; \mathrm{N}, 9.52 \%$ found: $\mathrm{C}, 73.42$; $H, 6.12 ; N, 9.50 \%$. 


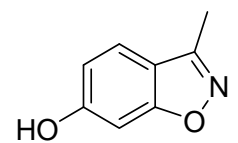

3-Methyl-6-hydroxy-1,2-benzisoxazole (7) ${ }^{1,2}$

Yield: 254 mg (86\%); colorless liquid. IR (KBr): 3258, 3080, 2947, 2930, 1635, 1610, 1566, 1521, 1312, 1138, $656 \mathrm{~cm}^{-1} .{ }^{1} \mathrm{H}$ NMR (300MHz, DM SO- $\left.d_{6}\right): \delta=2.38\left(\mathrm{~s}, 3 \mathrm{H}, \mathrm{CH}_{3}\right), 4.82(\mathrm{~s}, 1 \mathrm{H}, \mathrm{OH}), 6.89$ $(\mathrm{m}, 2 \mathrm{H}), 7.12(\mathrm{~m}, 1 \mathrm{H}) .{ }^{13} \mathrm{C}$ NMR $\left(75 \mathrm{MHz}, \mathrm{DMSO}-d_{6}\right): \delta=159.5,154.2,149.7,136.3,129.2,126.7$, 118.4, 18.8. GC-MS: m/z $149[\mathrm{M}]^{+}$. Anal. Calc. For $\mathrm{C}_{8} \mathrm{H}_{7} \mathrm{NO}_{2}$ : C, 64.42; H, 4.73; N, 9.39\% found: C, $64.40 ; \mathrm{H}, 4.71 ; \mathrm{N}, 9.38 \%$.

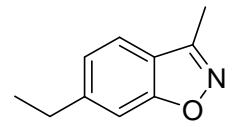

\section{6-Ethyl-3-methyl-1,2-benzisoxazole (8) ${ }^{1}$}

Yield: 292 mg (91\%); pale yellow liquid. IR (KBr): 3060, 3022, 2960, 1620, 1542, 1423, 1325, 1266, 750, $722 \mathrm{~cm}^{-1} .{ }^{1} \mathrm{H}$ NMR (300MHz, DMSO-d $\left.d_{6}\right): \delta=1.32\left(\mathrm{t}, 3 \mathrm{H}, \mathrm{CH}_{3}\right), 2.36\left(\mathrm{~s}, 3 \mathrm{H}, \mathrm{CH}_{3}\right), 2.52$ $\left(\mathrm{q}, 2 \mathrm{H}, \mathrm{CH}_{2}\right), 7.14(\mathrm{~m}, 3 \mathrm{H}) .{ }^{13} \mathrm{C}$ NMR $\left(75 \mathrm{MHz}, \mathrm{DMSO}-d_{6}\right): \delta=162.3,152.1,131.8,124.1,122.4$, 121.0, 110.2, 34.6, 16.5, 14.7. GC-MS: m/z $161[\mathrm{M}]^{+}$. Anal. Calc. For $\mathrm{C}_{10} \mathrm{H}_{11} \mathrm{NO}$ : C, 74.51; H, 6.88; N, 8.69\% found: C, 74.50; H, 6.86; N, $8.70 \%$.

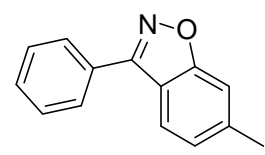

\section{6-Methyl-3-phenyl-1,2-benzisoxazole (9) ${ }^{1}$}

Yield: 365 mg (82\%); low melting colorless solid. IR (KBr): 3060, 3029, 2953, 2926, 2864, 2805, $1628,1601,1577,1549,1489,1417,1260,985,695 \mathrm{~cm}^{-1} .{ }^{1} \mathrm{H}$ NMR $(300 \mathrm{MHz}$, DMSO-d 6 ): $\delta=2.40$ $\left(\mathrm{s}, 3 \mathrm{H}, \mathrm{CH}_{3}\right), 7.10(\mathrm{~m}, 2 \mathrm{H}), 7.20(\mathrm{~m}, 1 \mathrm{H}), 7.30(\mathrm{~m}, 2 \mathrm{H}), 7.36(\mathrm{~m}, 1 \mathrm{H}), 7.52(\mathrm{~m}, 2 \mathrm{H}) .{ }^{13} \mathrm{C} N M R$ $\left(75 \mathrm{MHz}, \mathrm{DMSO}-d_{6}\right): \delta=162.3,154.2,142.3,135.4,129.3,127.4,127.2,125.8,124.6,124.1$, 122.1, 118.2, 112.7, 18.8. GC-MS: m/z 209 [M] ${ }^{+}$. Anal. Calc. For $\mathrm{C}_{14} \mathrm{H}_{11} \mathrm{NO}: \mathrm{C}, 80.36 ; \mathrm{H}, 5.30 ; \mathrm{N}$, 6.69\% found: C, 80.32; H, 5.25; N, $6.67 \%$. 


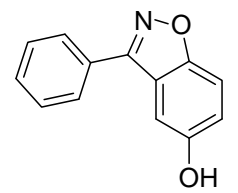

\section{6-Hydroxy-3-phenyl-1,2-benzisoxazole (10) ${ }^{1}$}

Yield: 372 mg (85\%); low melting colorless solid. IR (KBr): 3158, 3027, 2950, 2935, 2860, 2815, $1618,1622,1560,1552,1482,1411,1260,945,688 \mathrm{~cm}^{-1} .{ }^{1} \mathrm{H}$ NMR $\left(300 \mathrm{MHz}, \mathrm{DMSO}-d_{6}\right): \delta=4.86$ $(\mathrm{s}, 1 \mathrm{H}, \mathrm{OH}), 6.78(\mathrm{~m}, 2 \mathrm{H}), 7.24(\mathrm{~m}, 2 \mathrm{H}), 7.38(\mathrm{~m}, 2 \mathrm{H}), 7.56(\mathrm{~m}, 2 \mathrm{H}) .{ }^{13} \mathrm{C}$ NMR $\left(75 \mathrm{MHz}, \mathrm{DMSO}-d_{6}\right)$ : $\delta=160.3,152.2,140.4,135.6,129.0,128.5,127.2,125.8,124.6,122.1,122.0,118.4,112.1$. GCMS: m/z $211[\mathrm{M}]^{+}$. Anal. Calc. For $\mathrm{C}_{13} \mathrm{H}_{9} \mathrm{NO}_{2}$ : C, 73.92; $\mathrm{H}, 4.29 ; \mathrm{N}, 6.63 \%$ found: $\mathrm{C}, 73.91 ; \mathrm{H}, 4.29$; N, $6.62 \%$.

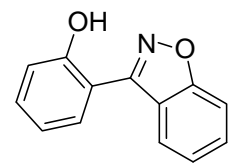

\section{3-(2'-Hydroxy) phenyl-1,2-benzisoxazole (11) ${ }^{1}$}

Yield: 360 mg (83\%); colorless solid. IR (KBr): 3220, 3022, 2968, 2948, 2860, 2814, 1618, 1622, 1537, 1512, 1487, 1422, 1260, $688 \mathrm{~cm}^{-1} .{ }^{1} \mathrm{H}$ NMR (300MHz, DMSO-d 6 ): $\delta=5.12(\mathrm{~s}, 1 \mathrm{H}, \mathrm{OH}), 6.89$ $(\mathrm{m}, 2 \mathrm{H}), 7.22(\mathrm{~m}, 2 \mathrm{H}), 7.44(\mathrm{~m}, 4 \mathrm{H}) .{ }^{13} \mathrm{C}$ NMR $\left(75 \mathrm{MHz}, \mathrm{DMSO}-d_{6}\right): \delta=161.5,151.2,142.4,136.3$, 129.4, 128.1, 127.4, 126.8, 125.3, 122.1, 121.7, 118.3, 112.0. GC-MS: m/z $211[\mathrm{M}]^{+}$. Anal. Calc. For $\mathrm{C}_{13} \mathrm{H}_{9} \mathrm{NO}_{2}$ : C, 73.92; $\mathrm{H}, 4.29 ; \mathrm{N}, 6.63 \%$ found: $\mathrm{C}, 73.90 ; \mathrm{H}, 4.28 ; \mathrm{N}, 6.61 \%$.

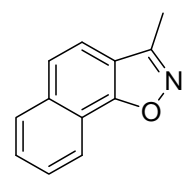

\section{3-Methylnaphtho[1,2-d]isoxazole (12) ${ }^{1,3}$}

Yield: 350 mg (94\%); colorless solid. IR (KBr): 3066, 3028, 2958, 1620, 1542, 1421, 1315, 1250, 850, $728 \mathrm{~cm}^{-1} .{ }^{1} \mathrm{H}$ NMR $\left(300 \mathrm{MHz}, \mathrm{DMSO}-d_{6}\right): \delta=2.35\left(\mathrm{~s}, 3 \mathrm{H}, \mathrm{CH}_{3}\right), 7.18(\mathrm{~m}, 2 \mathrm{H}), 7.34(\mathrm{~m}, 2 \mathrm{H})$, $7.40(\mathrm{~m}, 2 \mathrm{H}) .{ }^{13} \mathrm{C}$ NMR $\left(75 \mathrm{MHz}\right.$, DMSO- $\left.d_{6}\right): \delta=162.0,153.3,141.4,135.4,129.3,128.3,128.1$, 124.1, 121.8, 118.4, 112.5, 18.3. GC-MS: m/z $183[\mathrm{M}]^{+}$. Anal. Calc. For $\mathrm{C}_{12} \mathrm{H}_{9} \mathrm{NO}$ : C, 78.67; $\mathrm{H}$, $4.95 ; \mathrm{N}, 7.65 \%$ found: $\mathrm{C}, 78.64 ; \mathrm{H}, 4.90 ; \mathrm{N}, 7.62 \%$. 
Characterization data of various substituted isoxazolo and isothiazolo quinoline derivatives

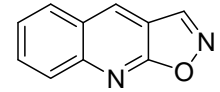

\section{Isoxazolo(5,4-b)quinoline (13) $5,6,7$}

Yield: $320 \mathrm{mg}$ (82\%); colorless solid; mp 188-190 C. IR (KBr): 3043, 2954, 1630, 1600, 1551, $1490,1442,1406,1324,1266,1177,846,747,689 \mathrm{~cm}^{-1} .{ }^{1} \mathrm{H}$ NMR $\left(300 \mathrm{MHz}, \mathrm{DMSO}-d_{6}\right): \delta=6.28$ $(\mathrm{s}, 1 \mathrm{H}), 7.30(\mathrm{~m}, 1 \mathrm{H}), 7.49(\mathrm{~m}, 1 \mathrm{H}), 7.72(\mathrm{~m}, 1 \mathrm{H}), 8.06(\mathrm{~s}, 1 \mathrm{H}), 8.18(\mathrm{~m}, 1 \mathrm{H}) .{ }^{13} \mathrm{C} N M R(75 M H z$, DMSO- $\left.d_{6}\right): \delta=151.2,148.5,143.3,139.1,129.3,128.9,128.1,127.7,122.4,120.4$. GC-MS: $\mathrm{m} / \mathrm{z}$ $170[\mathrm{M}]^{+}$. Anal. Calc. For $\mathrm{C}_{10} \mathrm{H}_{6} \mathrm{~N}_{2} \mathrm{O}: \mathrm{C}, 70.58 ; \mathrm{H}, 3.55 ; \mathrm{N}, 16.46 \%$ found: $\mathrm{C}, 70.55 ; \mathrm{H}, 3.51 ; \mathrm{N}$, $16.42 \%$.

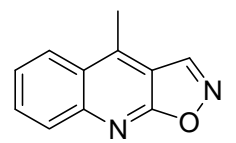

\section{4-Methyl-isoxazolo(5,4-b)quinoline $(14)^{5,6,7}$}

Yield: 368 mg (92\%); pale yellow solid; mp 198-199 C. IR (KBr): 3148, 3022, 2950, 2919, 1620, $1533,1453,1417,1260,1216,1028,822,689 \mathrm{~cm}^{-1} .{ }^{1} \mathrm{H}$ NMR $\left(300 \mathrm{MHz}, \mathrm{DMSO}-d_{6}\right): \delta=2.38(\mathrm{~s}$, $3 \mathrm{H}, \mathrm{Me}), 6.52(\mathrm{~s}, 1 \mathrm{H}), 7.38(\mathrm{~m}, 1 \mathrm{H}), 7.64(\mathrm{~m}, 1 \mathrm{H}), 7.80(\mathrm{~m}, 1 \mathrm{H}), 8.46(\mathrm{~m}, 1 \mathrm{H}) .{ }^{13} \mathrm{C} \mathrm{NMR}(75 \mathrm{MHz}$, DMSO- $\left.d_{6}\right): \delta=155.4,148.3,141.6,141.2,129.4,129.6,128.2,125.4,122.4,121.1$, 17.6. GC-MS: $\mathrm{m} / \mathrm{z} 184[\mathrm{M}]^{+}$. Anal. Calc. For $\mathrm{C}_{11} \mathrm{H}_{8} \mathrm{~N}_{2} \mathrm{O}: \mathrm{C}, 71.73 ; \mathrm{H}, 4.38 ; \mathrm{N}, 15.21 \%$ found: $\mathrm{C}, 71.71 ; \mathrm{H}, 4.36 ; \mathrm{N}$, $15.20 \%$.

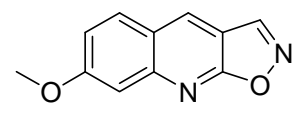

\section{7-Methoxy-isoxazolo(5,4-b)quinoline $(15)^{5,6,7}$}

Yield: $370 \mathrm{mg}$ (82\%). colorless solid; mp 190-192 C. IR (KBr): 3083, 3029, 2953, 2926, 2864, 2805, 1637, 1577, 1549, 1489, 1417, 1260, 985, $695 \mathrm{~cm}^{-1}$. ${ }^{1} \mathrm{H}$ NMR $(300 \mathrm{MHz}$, DMSO-d 6 ): $\delta=3.86$ $\left(\mathrm{s}, 3 \mathrm{H}, \mathrm{OCH}_{3}\right), 6.30(\mathrm{~s}, 1 \mathrm{H}), 7.30(\mathrm{~m}, 1 \mathrm{H}), 7.52(\mathrm{~m}, 1 \mathrm{H}), 7.70(\mathrm{~m}, 1 \mathrm{H}), 8.28(\mathrm{~m}, 1 \mathrm{H}) .{ }^{13} \mathrm{C} N M R$ $\left(75 \mathrm{MHz}, \mathrm{DMSO}-d_{6}\right): \delta=152.2,148.2,142.3,140.1,129.3,128.9,128.1,127.1,122.3,121.1$, 56.3. GC-MS: m/z $200[M]^{+}$. Anal. Calc. For $\mathrm{C}_{11} \mathrm{H}_{8} \mathrm{~N}_{2} \mathrm{O}_{2}$ : C, 66.00; H, 4.03; N, 13.99\% found: C, $65.97 ; H, 4.00 ; N, 13.96 \%$. 


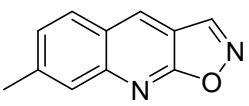

7-Methyl-isoxazolo(5,4-b)quinoline (16) ${ }^{5,6}$

Yield: $340 \mathrm{mg}$ (78\%); yellow solid; mp 193-195 C. IR (KBr): 3045, 3012, 2970, 2959, 1618, 1513, $1463,1407,1266,1246,1028,822,712 \mathrm{~cm}^{-1} .{ }^{1} \mathrm{H}$ NMR $\left(300 \mathrm{MHz}, \mathrm{DMSO}-d_{6}\right): \delta=2.42(\mathrm{~s}, 3 \mathrm{H}, \mathrm{Me})$, $6.48(\mathrm{~s}, 1 \mathrm{H}), 7.33(\mathrm{~m}, 1 \mathrm{H}), 7.58(\mathrm{~m}, 1 \mathrm{H}), 7.82(\mathrm{~m}, 1 \mathrm{H}), 8.40(\mathrm{~m}, 1 \mathrm{H}) .{ }^{13} \mathrm{C}$ NMR $\left(75 \mathrm{MHz}, \mathrm{DMSO}-d_{6}\right)$ : $\delta=156.1,149.3,141.3,141.1,129.3,128.7,128.2$, 126.4, 121.4, 121.1, 18.2. GC-MS: m/z 184 $[\mathrm{M}]^{+}$. Anal. Calc. For $\mathrm{C}_{11} \mathrm{H}_{8} \mathrm{~N}_{2} \mathrm{O}: \mathrm{C}, 71.73 ; \mathrm{H}, 4.38 ; \mathrm{N}, 15.21 \%$ found: $\mathrm{C}, 71.72 ; \mathrm{H}, 4.34 ; \mathrm{N}, 15.18 \%$.

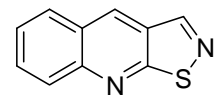

\section{Isothiazolo (5,4-b)quinoline (17) ${ }^{6,7}$}

Yield: $350 \mathrm{mg}$ (90\%); yellow solid; mp 168-170 ${ }^{\circ} \mathrm{C}$. IR (KBr): 3062, 2957, 2930, 2868, 1632, 1604, 1576, 1541, 1489, 1308, 1141, $696 \mathrm{~cm}^{-1} .{ }^{1} \mathrm{H}$ NMR $\left(300 \mathrm{MHz}, \mathrm{DMSO}-d_{6}\right): \delta=7.70(\mathrm{~m}, 1 \mathrm{H}), 7.84(\mathrm{~m}$, $2 \mathrm{H}), 7.98(\mathrm{~m}, 1 \mathrm{H}), 8.26(\mathrm{~s}, 1 \mathrm{H}), 8.57(\mathrm{~s}, 1 \mathrm{H}) .{ }^{13} \mathrm{C}$ NMR $\left(75 \mathrm{MHz}, \mathrm{DMSO}-d_{6}\right): \delta=160.4,151.2,146.6$, 136.6, 129.9, 128.1, 128.0, 127.3, 126.1, 119.3. GC-MS: m/z 186 [M] ${ }^{+}$. Anal. Calc. For $\mathrm{C}_{10} \mathrm{H}_{6} \mathrm{~N}_{2} \mathrm{~S}$ : C, 64.49; H, 3.25; N, $15.04 \%$ found: C, $64.40 ; H, 3.22 ; \mathrm{N}, 14.98 \%$.

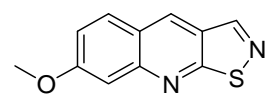

\section{7-Methoxy-isothiazolo(5,4-b)quinoline $(18)^{6,7}$}

Yield: $390 \mathrm{mg}$ (85\%); yellow solid; mp 222-224 ${ }^{\circ} \mathrm{C}$. IR (KBr): 3043, 2954, 1620, 1551, 1490, 1442, 1406, 1324, 1266, 1177, 846, 747, $689 \mathrm{~cm}^{-1} .{ }^{1} \mathrm{H}$ NMR $\left(300 \mathrm{MHz}, \mathrm{DMSO}-d_{6}\right): \delta=3.90(\mathrm{~s}, 3 \mathrm{H}$, $\left.\mathrm{OCH}_{3}\right), 7.41(\mathrm{~s}, 1 \mathrm{H}), 7.64(\mathrm{~d}, 1 \mathrm{H}, J=9.0 \mathrm{~Hz}), 7.85(\mathrm{~d}, 1 \mathrm{H}, J=9.0 \mathrm{~Hz}), 8.56(\mathrm{~s}, 1 \mathrm{H}), 8.87(\mathrm{~s}, 1 \mathrm{H}) .{ }^{13} \mathrm{C}$ NMR $\left(75 \mathrm{MHz}, \mathrm{DMSO}-d_{6}\right): \delta=162.4,159.1,150.7,146.6,141.8,135.1,128.3,127.4,126.4$, 125.2, 119.3, 55.6. GC-MS: m/z 216 [M] ${ }^{+}$. Anal. Calc. For $\mathrm{C}_{11} \mathrm{H}_{8} \mathrm{~N}_{2} \mathrm{OS}: \mathrm{C}, 61.09 ; \mathrm{H}, 3.73 ; \mathrm{N}, 12.95 \%$ found: $C, 61.04 ; H, 3.71 ; N, 12.90 \%$. 


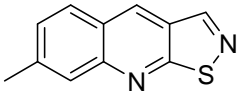

\section{7-Methyl-isothiazolo(5,4-b)quinoline (19) ${ }^{7}$}

Yield: $388 \mathrm{mg}$ (90\%); light brown solid; $\mathrm{mp} \mathrm{188-190}{ }^{\circ} \mathrm{C}$; IR (KBr): 3068, 3017, 2958, 2931, 2871, $1628,1547,1490,1308,846,749 \mathrm{~cm}^{-1}$. ${ }^{1} \mathrm{H}$ NMR $\left(300 \mathrm{MHz}, \mathrm{DMSO}-d_{6}\right): \delta=2.58\left(\mathrm{~s}, 3 \mathrm{H}, \mathrm{C} 7-\mathrm{CH}_{3}\right)$, $7.51(\mathrm{~s}, 1 \mathrm{H}), 7.68(\mathrm{~d}, 1 \mathrm{H}, J=9.0 \mathrm{~Hz}), 7.94(\mathrm{~d}, 1 \mathrm{H}, J=9.0 \mathrm{~Hz}), 8.72(\mathrm{~s}, 1 \mathrm{H}), 8.98(\mathrm{~s}, 1 \mathrm{H}) .{ }^{13} \mathrm{C} \mathrm{NMR}$ $\left(75 \mathrm{MHz}, \mathrm{DMSO}-d_{6}\right): \delta=161.5,158.8,149.7,146.6,140.0,136.9,128.3,128.1,127.1,126.6$, 118.4, 18.4. GC-MS: m/z 200 [M] ${ }^{+}$. Anal. Calc. For $\mathrm{C}_{11} \mathrm{H}_{8} \mathrm{~N}_{2} \mathrm{~S}: \mathrm{C}, 65.98 ; \mathrm{H}, 4.03 ; \mathrm{N}, 13.99 \%$ found: C, $65.92 ; H, 3.98 ; N, 13.96 \%$.

\section{REFERENCES}

1. Iranpoor, N.; Firouzabadi, H.; Nowrouzi, N. Tetrahedron Lett. 2006, 47, 8247.

2. Udd, S.; Jokela, R.; Franzén, R.; Tois, J. Tetrahedron Lett. 2010, 51, 1030.

3. Searcey, M.; Grewal, S. S.; Madeo, F.; Petros, G.T. Tetrahedron Lett. 2003, 44, 6745.

4. Yan, P.; Batamack, P.; Surya Prakash, G. K.; Olah, G. A. Catal Lett. 2005,101, 141

5. Laaman, S. M.; Meth-Cohn, O.; Recs, C.W. Synthesis 1999, 757.

6. Choi, J. H.; Choi, E. B.; Pak, C.S. Synlett 2003, 166.

7. Zlotin, S. G.; Kislitsin, P. G.; Podgursky, A. I.; Samet, A. V.; Buchana, A. C.; Gakh, A. A. J. Org. Chem. 2000, 65, 8439. 